\title{
Efecto del ácido indolbutírico (AIB) y edad de las estacas en el enraizamiento de Myrceugenia exsucca
}

\author{
Effect of indole butyric acid (IBA) and cutting age \\ on rooting in Myrceugenia exsucca
}

\author{
Marisol Muñoz *a, Ricardo Molina ${ }^{b}$ \\ *Autor de correspondencia: a Universidad de Talca, Facultad de Ciencias Forestales, Departamento de Producción Forestal, \\ Avenida Lircay s/n, Talca, Chile, tel.: 56712200 368,mmunoz@utalca.cl \\ ${ }^{\mathrm{b}}$ Corporación Nacional Forestal, Provincial Linares, Chile.
}

\section{SUMMARY}

Among the species that live in wetlands Myrceugenia exsucca is a highly important tree for the protection of areas with frequent flooding, edges in watercourses and streams. This study therefore is aimed at evaluating the effect of the application of indole butyric acid (IBA) and the age of the material on rooting in the $M$. exsucca cuttings. A completely randomized design, factorial, with two types of cutting: juvenile and mature, and six concentrations of IBA $\left(0,1,000,2,000,3,000,4,000\right.$ and 5,000 $\left.\mathrm{mg} \mathrm{L}^{-1}\right) \mathrm{were}$ tested. Six replications; each replicate consisted of 10 cuttings. The trial was conducted in a warm bed, with temperatures between 20 and $25^{\circ} \mathrm{C}$. The variables studied were: survival (\%), callus formation (\%), rooting (\%), root number, root length (cm) and new leaves. The age of cuttings had significant effects on all variables. The application of IBA had significant effects on the survival and rooting cuttings. The best results, over $85 \%$ of rooting were obtained with cuttings from juvenile material and with the two higher doses of IBA.

Key words: Myrceugenia exsucca, vegetative propagation, swamp forest.

\section{RESUMEN}

Entre las especies que habitan humedales se encuentra Myrceugenia exsucca, árbol de gran importancia para la protección de áreas con frecuente anegamiento, orillas en cursos de agua y quebradas. El objetivo del estudio fue evaluar el enraizamiento de $M$. exsucca al usar dos tipos de esquejes y aplicar distintas concentraciones de ácido indolbutírico (AIB). Se usó un diseño completamente aleatorizado, factorial, con estacas juveniles y maduras y se probaron diferentes concentraciones de AIB (0, 1.000, 2.000, 3.000, 4.000 y $\left.5.000 \mathrm{mg} \mathrm{L}^{-1}\right)$. Se hicieron seis repeticiones, cada una de ellas con 10 estacas. El ensayo se realizó en cama caliente, con temperaturas entre 20 y $25{ }^{\circ} \mathrm{C}$. Las variables estudiadas fueron: sobrevivencia (\%), estacas con callo (\%), enraizamiento (\%), número de raíces, longitud de raíces (cm) y número de hojas nuevas. La edad de la estaca influyó significativamente en todas las variables evaluadas. La aplicación de AIB afectó significativamente la sobrevivencia y enraizamiento de esquejes. Los mejores resultados, sobre $85 \%$ de estacas enraizadas, se lograron con esquejes provenientes de material juvenil y con las dos mayores dosis de AIB.

Palabras clave: Myrceugenia exsucca, propagación vegetativa, bosques higrófilos.

\section{INTRODUCCIÓN}

En riberas de ríos y lagos, y especialmente en quebradas, los bosques con Myrtaceas brindan protección contra la erosión (Ramírez et al. 1995). Myrceugenia exsucca (de Candolle) Berg forma parte de estos bosques pantanosos, perennifolios, que se encuentran en sectores con anegamiento estacional y aunque el hábitat no es apropiado para la agricultura estos ecosistemas son impactados por acciones antrópicas, reduciendo su área de distribución y fragmentando los rodales (Ramírez et al. 1995). Estos bosques higrófilos tienen gran importancia, tanto por su riqueza florística, como por constituir hábitat para gran diversidad de plantas y fauna, especialmente aves migratorias (Hauenstein et al. 2002, 2014).

Myrceugenia exsucca se distribuye en Chile entre $31^{\circ}$ y $43^{\circ} 30^{\prime}$ de latitud sur (Donoso 2006), y en Argentina se encuentra en Los Andes de Neuquén y Río Negro hasta Chubut (Landrum 1988); es nativa de ambos países (Latsague et al. 2010). Crece en diferentes altitudes, preferentemente en terrenos planos, siempre asociada a suelos inundados y cursos de agua, con sus raíces totalmente sumergidas en agua (Donoso 2006, Eyzaguirre 2010). Este árbol puede crecer entre 10 y $12 \mathrm{~m}$ de altura (Montenegro 2000) y $50 \mathrm{~cm}$ de diámetro a la altura del pecho, el tronco presenta ranuras o estrías longitudinales que dan la impresión de varios 
troncos creciendo juntos (Donoso 2006, Eyzaguirre 2010). Tiene usos apícolas, medicinales y ornamentales (Montenegro 2000). Su madera, aunque propensa a la pudrición y ataque de hongos, especialmente en el duramen, es fácil de trabajar, cepillar y encolar y dependiendo de las medidas que se consigan es apta para variados usos, como puertas, ventanas, mueblería, etc. (Witte y Hall 2004). Está catalogada como especie vulnerable para la región de Coquimbo (Squeo et al. 2001) y en peligro de extinción para la región de O'Higgins (Serey et al. 2007). Hauenstein et al. (2014) evaluaron el bosque pantanoso de temo-pitra (Blepharocalyo-Myrcengenietum exsuccae), ubicado entre las coordenadas $38^{\circ} 30^{\prime}-39^{\circ} 30^{\prime} \mathrm{S}$ y $72^{\circ} 45^{\prime}-73^{\circ} 30^{\prime} \mathrm{O}$, y concluyen que el estado promedio de conservación de esos rodales corresponde a degradado, además señalan que la supervivencia e integridad de estos bosques se relaciona con la capacidad y estrategia reproductiva de las especies arbóreas.

Para tareas de restauración, enriquecimiento o conservación ex situ se necesita contar con plantas, producidas por métodos sexuados o vegetativos. No hay evaluaciones sobre la producción de frutos de $M$. exsucca, según Donoso (2006) parece que no posee abundante floración y fructificación. Sus semillas no se pueden almacenar por largos periodos porque son recalcitrantes (Saldías y Veloso 2014).

En relación a su propagación vegetativa, estudios realizados por Latsague et al. (2010) con estacas semileñosas, provenientes de la sección media de las copas de árboles con edades estimadas en 50 años, colectadas en abril y mantenidas durante cuatro meses en cama caliente, se obtiene un máximo de $26,7 \%$ de estacas enraizadas al utilizar $2.000 \mathrm{mg} \mathrm{L}^{-1}$ de ácido indolbutírico (AIB). Estos autores concluyen que los bajos porcentajes de enraizamiento obtenidos podrían relacionarse con el tipo de estacas, época de recolección y concentración hormonal. A base de esta información, para este trabajo, se considera como hipótesis que, concentraciones sobre $3.000 \mathrm{mg} \mathrm{L}^{-1}$ de AIB mejoran la formación de raíces en estacas juveniles de $M$. exsucca. El objetivo fue comparar el efecto de distintas dosis de ácido indolbutírico (AIB) en el enraizamiento de estacas provenientes de material juvenil y adulto de $M$. exsucca.

\section{MÉTODOS}

El material fue colectado en una población con individuos adultos de M. exsucca, en la región de O'Higgins, en una quebrada en condiciones de anegamiento $\left(34^{\circ} 13^{\prime}\right.$ $43,53^{\prime \prime} \mathrm{S}$ y $\left.71^{\circ} 55^{\prime} 33,61^{\prime \prime} \mathrm{O}\right)$. La edad de los árboles no fue determinada, pero sus diámetros a $1,30 \mathrm{~cm}$ del suelo, superaban los $20 \mathrm{~cm}$ y con alturas entre 4 y $9 \mathrm{~m}$, por lo que se suponen adultos.

En el mes de agosto se colectó material de dos tipos, el juvenil proveniente de los rebrotes originados a nivel del tocón de los árboles y el adulto se obtuvo desde la copa de los individuos maduros. Se cortaron estacas semileñosas de 25 a $30 \mathrm{~cm}$ de largo, cuyas bases fueron envueltas en toallas de papel húmedo para evitar la deshidratación du- rante el traslado. En laboratorio, se uniformaron a un tamaño de $15 \mathrm{~cm}$ de largo y se les extrajeron las hojas de los dos tercios inferiores. Posteriormente, todo el material se lavó en agua corriente y se sometió a un baño de desinfección, con una solución de Captan ${ }^{\circledR}(0,1 \%)$ durante 10 minutos. A continuación, se realizó un corte recto en la base de cada estaca y se colocaron por cinco segundos en las soluciones con las distintas dosis de AIB. Se usaron contenedores de plástico transparente, de $500 \mathrm{~cm}^{3}$, con perlita como sustrato y se ubicaron en cama caliente con temperaturas entre 20 y $25^{\circ} \mathrm{C}$. Se regó a través de microaspersores y la humedad relativa se mantuvo sobre $70 \%$. Semanalmente se aplicó el fungicida Captan ${ }^{\circledR}(0,1 \%)$ y fertilizante foliar Phyllum ${ }^{\circledR}(0,01 \%)$, ya que los nutrientes minerales son lixiviados con la neblina de riego, lo que contribuye a una deficiencia foliar (Hartmann y Kester 2011).

Se usó un diseño factorial, completamente aleatorizado, con seis niveles para las concentraciones de AIB $\left(0,1.000,2.000,3.000,4.000\right.$ y $\left.5.000 \mathrm{mg} \mathrm{L}^{-1}\right)$ y dos edades para las estacas (juvenil y adulto). Para cada tratamiento se realizaron seis repeticiones de 10 estacas cada una.

Después de cuatro meses se evaluó la sobrevivencia (\%), estacas con callo (\%), enraizamiento (\%), número de raíces primarias, longitud de raíces primarias $(\mathrm{cm})$ medida como la sumatoria del largo de raíces primarias y número de hojas nuevas.

Previo al análisis estadístico, los datos porcentuales fueron transformados mediante arcoseno $\sqrt{x / \%}$. Para número de raíces se realizó transformación $\sqrt{x+1}$ y para el resto de las variables se usó $\sqrt{x}$, donde $x$ es el dato de conteo (Montgomery 1991). Los datos transformados fueron sometidos a análisis de varianza, usando el programa estadístico Statgraphics Centurión XV. Se usó la prueba de Tukey al $5 \%$ para determinar diferencias entre las medias de los tratamientos.

\section{RESULTADOS}

La edad de la estaca influyó significativamente en todas las variables evaluadas (cuadro 1). Se obtuvieron mejores resultados en estacas juveniles para sobrevivencia, formación de callo, enraizamiento y número de hojas nuevas. En las estacas de material adulto fue mayor el número de raíces y la longitud de estas.

La aplicación de AIB afectó significativamente solo la sobrevivencia $(P=0,0192)$ y enraizamiento de las estacas $(P=0,0255)$. En ambos casos, el mejor resultado $(81,7 \%)$ se logró con la aplicación de $5.000 \mathrm{mg} \mathrm{L}^{-1}$ de AIB.

La interacción de edad y hormona influyó significativamente en el enraizamiento de las estacas $(P=0,0339)$. Se observa que en estacas juveniles hay tendencia a aumentar el enraizamiento con la aplicación de mayores dosis de AIB, desde $66,7 \%$ (testigo) hasta $88,3 \%$ si se adiciona 5.000 $\mathrm{mg} \mathrm{L}^{-1}$ de AIB. En estacas adultas no hay claridad en este aspecto, obteniendo el menor enraizamiento con $3.000 \mathrm{mg} \mathrm{L}^{-1}$ y los valores más altos con 1.000 y 5.000 de mg L ${ }^{-1}$ AIB. 


\section{DISCUSIÓN}

Los porcentajes de estacas enraizadas, obtenidos en este estudio, superan los reportados anteriormente para M. exsucca (Latsague et al. 2010) y para otras mirtáceas chilenas. Saldías y Velozo (2014) lograron 33,3\% de enraizamiento en esquejes de Myrcianthes coquimbensis (Barnéoud) Landrum et Grifo cuando aplicaron $3.000 \mathrm{mg} \mathrm{L}^{-1} \mathrm{de}$ AIB, Latsague et al. (2010) obtuvieron $41 \%$ con $2.000 \mathrm{mg}$ $\mathrm{L}^{-1}$ de AIB en Blepharocalyx cruckshanksii (Hooker et Ar- nott) Niedenzu, Rodríguez et al. (2015) consiguieron $85 \%$ con $4.000 \mathrm{mg} \mathrm{L}^{-1}$ de AIB en microtallos de Ugni molinae Turcz.

Las estacas de M. exsucca provenientes de material juvenil enraízan mejor que las de material adulto, situación también descrita para Taxus globosa Schltdl. (Muñoz et al. 2009), Dalbergia melanoxylon Guill. et Perr. (Amri et al. 2010), Abies religiosa (Kunth) Schltdl. et Cham. (Castillo et al. 2013), Prosopis alba Grisebach (De Souza et al. 2014). Lo anterior posiblemente ocurre porque con la edad

Cuadro 1. Efecto del tipo de esqueje y concentración de AIB en la capacidad de enraizamiento de estacas de M. exsucca. Effect of type of stem cuttings and concentration of IBA in the capacity of rooting of cuttings of $M$. exsucca.

\begin{tabular}{|c|c|c|c|c|c|c|c|c|c|c|c|}
\hline Edad & $\begin{array}{c}\text { Sobreviven } \\
(\%)\end{array}$ & & $\begin{array}{c}\text { Callo } \\
(\%)\end{array}$ & & $\begin{array}{c}\text { Enraizamiento } \\
(\%)\end{array}$ & $\begin{array}{l}\mathrm{N}^{\mathrm{o}} \\
\text { raíces }\end{array}$ & & $\begin{array}{l}\text { Longitud de } \\
\text { Raíces }(\mathrm{cm})\end{array}$ & & $\begin{array}{r}\mathrm{N}^{\circ} \text { hojas } \\
\text { nuevas }\end{array}$ & \\
\hline Juvenil & $75,8 \pm 16,3$ & a & $83,6 \pm 4,9$ & $\mathrm{a}$ & $76,7 \pm 19,7 \quad a$ & $5,9 \pm 0,3$ & & $24,9 \pm 1,4$ & & $5,8 \pm 0,7$ & $\mathrm{a}$ \\
\hline Adulto & $66,1 \pm 16,8$ & $\mathrm{~b}$ & $63,3 \pm 3,0$ & $\mathrm{~b}$ & $64,4 \pm 16,4$ & $6,9 \pm 0,8$ & $\mathrm{~b}$ & $29,0 \pm 2,9$ & $\mathrm{~b}$ & $1,8 \pm 0,1$ & $\mathrm{~b}$ \\
\hline Significación & $*$ & & * & & $*$ & $*$ & & $*$ & & $*$ & \\
\hline \multicolumn{12}{|l|}{ AIB mg L ${ }^{-1}$} \\
\hline 0 & $65,0 \pm 18,3$ & $a b$ & $73,3 \pm 15,6$ & $\mathrm{a}$ & $65,8 \pm 17,3 \quad a b$ & $5,6 \pm 1,9$ & & $23,8 \pm 5,6$ & & $5,8 \pm 2,9$ & $\mathrm{a}$ \\
\hline 1.000 & $75,0 \pm 10,9$ & $a b$ & $79,2 \pm 9,0$ & $\mathrm{a}$ & $73,3 \pm 13,0 \quad a b$ & $6,5 \pm 2,0$ & & $28,3 \pm 9,4$ & & $4,0 \pm 2,2$ & $\mathrm{a}$ \\
\hline 2.000 & $68,3 \pm 20,8$ & $a b$ & $69,2 \pm 20,2$ & $\mathrm{a}$ & $67,5 \pm 20,5 \quad a b$ & $6,3 \pm 1,5$ & $\mathrm{a}$ & $27,0 \pm 6,4$ & $\mathrm{a}$ & $4,2 \pm 2,1$ & $\mathrm{a}$ \\
\hline 3.000 & $63,3 \pm 16,7$ & $\mathrm{a}$ & $65,0 \pm 20,2$ & $\mathrm{a}$ & $62,5 \pm 17,6 \quad a$ & $6,7 \pm 1,9$ & $\mathrm{a}$ & $28,5 \pm 7,0$ & $\mathrm{a}$ & $4,1 \pm 2,0$ & $\mathrm{a}$ \\
\hline 4.000 & $72,5 \pm 15,4$ & $\mathrm{ab}$ & $75,0 \pm 18,8$ & $\mathrm{a}$ & $72,5 \pm 18,2 \quad a b$ & $6,1 \pm 1,6$ & $\mathrm{a}$ & $25,1 \pm 7,0$ & & $4,6 \pm 2,3$ & $\mathrm{a}$ \\
\hline 5.000 & $81,7 \pm 15,3$ & $\mathrm{~b}$ & $79,2 \pm 14,4$ & $\mathrm{a}$ & $81,7 \pm 15,3$ & $6,8 \pm 1,0$ & $\mathrm{a}$ & $27,8 \pm 5,0$ & $\mathrm{a}$ & $4,4 \pm 2,3$ & $\mathrm{a}$ \\
\hline Significación & $*$ & & ns & & $*$ & ns & & ns & & ns & \\
\hline \multicolumn{12}{|l|}{$\begin{array}{c}\text { Edad x AIB } \\
\mathrm{mg} \mathrm{L}^{-1}\end{array}$} \\
\hline Juvenil - 0 & $68,3 \pm 19,4$ & a & $83,3 \pm 10,3$ & $\mathrm{a}$ & $66,7 \pm 15,1 \quad a b c$ & $5,0 \pm 1,0$ & $\mathrm{a}$ & $22,1 \pm 4,0$ & $\mathrm{a}$ & $6,9 \pm 2,7$ & $\mathrm{a}$ \\
\hline Juvenil - 1000 & $70,0 \pm 12,6$ & a & $78,3 \pm 11,7$ & $\mathrm{a}$ & $66,7 \pm 20,7 \mathrm{abc}$ & $5,5 \pm 0,8$ & $\mathrm{a}$ & $22,7 \pm 4,4$ & $\mathrm{a}$ & $5,5 \pm 1,5$ & $\mathrm{a}$ \\
\hline Juvenil - 2000 & $76,7 \pm 21,6$ & $\mathrm{a}$ & $80,0 \pm 17,9$ & $\mathrm{a}$ & $76,7 \pm 8,2 \quad a b c$ & $5,8 \pm 1,2$ & $\mathrm{a}$ & $27,3 \pm 6,5$ & a & $5,5 \pm 1,4$ & $\mathrm{a}$ \\
\hline Juvenil - 3000 & $75,0 \pm 8,4$ & $\mathrm{a}$ & $81,7 \pm 9,8$ & $\mathrm{a}$ & $76,7 \pm 12,2 \quad a b c$ & $6,8 \pm 1,7$ & $\mathrm{a}$ & $29,8 \pm 6,6$ & $\mathrm{a}$ & $5,1 \pm 1,2$ & $\mathrm{a}$ \\
\hline Juvenil - 4000 & $76,7 \pm 19,7$ & $\mathrm{a}$ & $90,0 \pm 6,3$ & $\mathrm{a}$ & $85,0 \pm 9,8$ & $5,3 \pm 0,8$ & $\mathrm{a}$ & $21,6 \pm 4,0$ & $\mathrm{a}$ & $5,9 \pm 1,0$ & $\mathrm{a}$ \\
\hline Juvenil - 5000 & $88,3 \pm 9,8$ & $\mathrm{a}$ & $88,3 \pm 9,8$ & $\mathrm{a}$ & $88,3 \pm 3,8$ & $6,6 \pm 1,1$ & $\mathrm{a}$ & $25,5 \pm 4,6$ & $\mathrm{a}$ & $6,0 \pm 0,7$ & $\mathrm{a}$ \\
\hline Adulto - 0 & $61,7 \pm 18,3$ & a & $63,3 \pm 13,7$ & $\mathrm{a}$ & $65,0 \pm 6,3$ & $6,2 \pm 2,5$ & $\mathrm{a}$ & $25,6 \pm 7,0$ & $\mathrm{a}$ & $2,5 \pm 1,3$ & $\mathrm{a}$ \\
\hline Adulto -1000 & $80,0 \pm 6,3$ & $\mathrm{a}$ & $80,0 \pm 6,3$ & $\mathrm{a}$ & $80,0 \pm 17,2$ abc & $7,3 \pm 2,4$ & $\mathrm{a}$ & $33,1 \pm 10,3$ & & $2,0 \pm 0,5$ & $\mathrm{a}$ \\
\hline Adulto -2000 & $60,0 \pm 17,9$ & $\mathrm{a}$ & $58,3 \pm 17,2$ & $\mathrm{a}$ & $58,3 \pm 11,7 \quad a b$ & $6,9 \pm 1,8$ & $\mathrm{a}$ & $26,6 \pm 6,7$ & $\mathrm{a}$ & $1,8 \pm 0,3$ & $\mathrm{a}$ \\
\hline Adulto -3000 & $51,7 \pm 14,7$ & a & $48,3 \pm 11,7$ & $\mathrm{a}$ & $48,3 \pm 14,1 \quad a$ & $6,7 \pm 2,3$ & $\mathrm{a}$ & $26,5 \pm 7,3$ & $\mathrm{a}$ & $1,8 \pm 0,4$ & $\mathrm{a}$ \\
\hline Adulto -4000 & $68,3 \pm 9,8$ & a & $60,0 \pm 14,1$ & $\mathrm{a}$ & $60,0 \pm 17,6 \quad a b$ & $7,2 \pm 1,6$ & $\mathrm{a}$ & $30,1 \pm 7,0$ & $\mathrm{a}$ & $1,6 \pm 0,4$ & $\mathrm{a}$ \\
\hline Adulto -5000 & $75,0 \pm 17,6$ & $\mathrm{a}$ & $70,0 \pm 12,6$ & $\mathrm{a}$ & $75,0 \pm 5,9$ & $7,1 \pm 1,0$ & $\mathrm{a}$ & $30,4 \pm 4,3$ & $\mathrm{a}$ & $1,7 \pm 0,2$ & $\mathrm{a}$ \\
\hline Significación & ns & & ns & & $*$ & ns & & ns & & ns & \\
\hline
\end{tabular}

Medias \pm desviación estándar. Nivel de significación (ns: no significativo; *: significativo $P<0,05$ ). Letras distintas en las columnas, dentro de cada factor indican diferencias significativas entre tratamientos con la prueba de Tukey.

Mean \pm standard deviation. Level of significance (ns: not significant; *: significant $P<0.05$ ). Different letters in the columns, within each factor, indicate significant differences among treatments with Tukey test. 
disminuyen compuestos fenólicos, que actúan como cofactores en la iniciación de raíces (Hartmann et al. 2011). El vigor de estacas juveniles de $M$. exsucca propicia la aparición de nuevas hojas, aumentando su número hasta cuatro veces en relación a las estacas maduras $(5,8$ versus $1,8)$, lo que podría favorecer la sobrevivencia y enraizamiento, ya que los carbohidratos transportados desde las hojas contribuyen al desarrollo de raíces adventicias (Hartmann et al. 2011). La importancia de las hojas se evidencia en la investigación realizada por Latsague et al. (2010) con B. cruckshanksii, cuyos bajos porcentajes de enraizamiento y sobrevivencia son atribuidos por estos autores a que la mayoría de las estacas perdieron sus hojas durante el ensayo. La ausencia total de foliolos en estacas de Litchi chinensis Sonn. generó la muerte de estas y la presencia de, al menos, un foliolo fue suficiente para tener promedios de sobrevivencia sobre $70 \%$ y con cuatro foliolos se aumentó a $95 \%$, alcanzando también el máximo enraizamiento de $60 \%$ (Alves et al. 2016).

La época de cosecha del material también es un factor que influye en el enraizamiento (Hartmann et al. 2011). Se recomienda otoño e invierno para Berberidopsis corallina Hook.f. (Uribe et al. 2011), diciembre para A. religiosa (Castillo et al. 2013). Pero el problema es complejo porque el momento no depende del calendario, se refiere a la condición fisiológica de la planta y es necesario investigar para encontrar el momento adecuado a cada especie (Wells 1985). El periodo durante la floración puede ser antagónico al enraizamiento, a veces se obtienen mejores resultados si se colectan las estacas antes o después de este proceso (Hartmann et al. 2011). En M. exsucca la floración varía entre enero a mayo, dependiendo de la localidad, y fructifica entre junio a septiembre (Rodríguez et al. 1983). Latsague et al. (2010) colectaron en periodo de floración y en este trabajo se colectó después de ese proceso, lo que también podría explicar las diferencias entre ambas experiencias, pero se requiere mayor investigación al respecto, considerando además que Donoso (2006) señala que esta especie "parece no florecer todos los años".

En ausencia de auxina se logran porcentajes satisfactorios de enraizamiento, tanto en estacas juveniles y maduras de $M$. exsucca, esto es posible porque las estacas poseen cierta cantidad de hormonas endógenas que pueden inhibir o estimular el desarrollo de raíces adventicias (Hartmann et al. 2011). En el material juvenil de algunas especies, los niveles endógenos son suficientes para inducir el enraizamiento, sin necesidad de aplicaciones exógenas, este comportamiento fue evidenciado por De Souza et al. (2014), quienes obtuvieron hasta un $100 \%$ de enraizamiento con mini estacas obtenidas de plantas de dos años de P. alba. A diferencia de los resultados obtenidos por Latsague et al. (2010), en este estudio se observa el efecto significativo de la auxina sobre el enraizamiento, siendo más evidente en estacas juveniles, situación también descrita para D. melanoxylon (Amri et al. 2010). Al aplicar AIB también se mejora la calidad del sistema radical, porque aumen- ta el número de raíces y la longitud de estas, aunque sin efectos significativos, este comportamiento coincide con lo reportado para U. molinae (Rodríguez et al. 2015) y B. corallina (Uribe et al. 2011).

En el caso de estacas maduras, se consiguen mejores valores para todas las variables evaluadas, con excepción del número de hojas nuevas, cuando se aplican $1.000 \mathrm{mg}$ $\mathrm{L}^{-1}$ de AIB. Al respecto, Alves et al. (2016) señalan que esta concentración de AIB ha permitido obtener respuestas satisfactorias, en varios estudios con estacas de especies frutales.

Finalmente, se validó la hipótesis planteada, ya que se consiguieron los mejores resultados de enraizamiento en estacas juveniles con dosis mayores a $3.000 \mathrm{mg} \mathrm{L}^{-1}$ de AIB, contribuyendo en el protocolo de propagación vegetativa para esta especie, sin embargo se recomienda seguir investigando sobre la fenología y su relación con el momento más adecuado para cosechar estacas.

\section{REFERENCIAS}

Alves E, J Guimarães, C Franco, A Martins. 2016. Number of leaflets on rooting of lychee herbaceous cuttings. Ciência Rural 46(6): 1003-1006.

Amri E, HVM Lyaruu, AS Nyomora, ZL Kanyeka. 2010. Vegetative propagation of African Blackwood (Dalbergia melanoxylon Guill. et Perr.): effects of age of donor plant, IBA treatment and cutting position on rooting ability of stem cuttings. New Forests 39:183-194.

Castillo D, M López, J López, V Cetina, T Hernández. 2013. Factores de influencia en el enraizamiento de estacas de Abies religiosa (Kunth) Schltdl. et Cham. Revista Chapingo. Serie Ciencias Forestales y del Ambiente 19(1):175-184.

De Souza J, A Bender, J Tivano, D Barroso, L Mroginski, A Vegetti, Felker P. 2014. Rooting of Prosopis alba mini-cuttings. New Forests 45(5): 745-752.

Donoso C. 2006. Myrceugenia exsucca (D.C.) Berg. In Donoso $\mathrm{C}$ ed. Especies arbóreas de los bosques templados de Chile y Argentina. Autoecología. Valdivia, Chile. Marisa Cuneo Ediciones. p. 375-380.

Eyzaguirre MT. 2010. Familia Myrtaceae Juss. en Chile. Chile natural, Fundación RA Philippi de estudios naturales, Chile. Consultado 12 ago. 2014. Disponible en http://fundacionraphilippi.cl/fundacion/t_chile_myrtaceae.html

Hartmann HT, DE Kester, FT Davies and RL Geneve. 2011. Plant propagation: Principles and Practice. Eighth edition. New Jersey, USA. Prentice Hall. 915 p.

Hauenstein E, M González, F Peña-Cortés, A Muñoz-Pedreros. 2002. Clasificación y caracterización de la flora y vegetación de los humedales de la costa de Toltén (IX Región, Chile). Gayana Botánica 59(2): 87-100.

Hauenstein E, M González, F Peña-Cortés, C Bertrán, J Tapia, L Vargas Chacoff, O Urrutia. 2014. Composición florística y evaluación de la degradación del bosque pantanoso costero de temu-pitra en la Región de la Araucanía, Chile. Gayana Botánica 71(1): 43-57.

Landrum L. 1988. The myrtle family (Myrtaceae) in Chile. Proceedings of California Academy Sciences 45(12): 277-317.

Latsague M, P Sáez Delgado, E Hauenstein Barra, F Peña-Cor- 
tés. 2010. Propagación vegetativa de Myrceugenia exsucca y Blepharocalyx cruckshanksii, especies dominantes del bosque pantanoso de la Depresión Intermedia de la región de La Araucanía, Chile. Bosque 31(3): 247-251.

Montgomery D. 1991. Diseño y análisis de experimentos. México DF, México. Grupo Editorial Iberoamericano. 589 p.

Montenegro G. 2000. Chile, Nuestra Flora Útil. Guía de Uso Apícola, Alimentario, Medicinal Folclórico, Artesanal y Ornamental. Colección en Agricultura. Santiago, Chile. Ediciones Universidad Católica de Chile. 267 p.

Muñoz L, J Vargas, J López, M Soto. 2009. Effect of cutting age and substrate temperature on rooting of Taxus globosa. New Forests 38(2): 187-196.

Ramírez C, C San Martín, J. San Martín. 1995. Estructura florística de los bosques pantanosos de Chile sur-central. pp. 215-234. In Armesto J, C Villagrán, M Arroyo eds. Ecología de los bosques nativos de Chile. Santiago, Chile. Editorial Universitaria. $470 \mathrm{p}$.

Rodríguez R, O Matthei, M Quezada. 1983. Flora Arbórea de Chile. pp. 215-216. Concepción, Chile. Editorial de la Universidad de Concepción. 408 p.

Rodríguez M, R Carrillo, M Chacón, N Hormazábal, J Tampe,
$\mathrm{R}$ Tighe. 2015. Enraizamiento in vitro y ex vitro de microtallos de Ugni molinae Turcz., una especie nativa de Chile. Gayana. Botánica, 72(1): 14-20.

Saldías G, J Velozo. 2014. Estudio de la propagación de $M y r$ cianthes coquimbensis (Barnéoud) Landrum et Grifo por semillas y esquejes. Gayana Botánica 71(1): 17-23.

Serey I, M Ricci, C Smith-Ramírez (Eds.) 2007. Libro Rojo de la Región de O’Higgins. Rancagua, Chile. Corporación Nacional Forestal - Universidad de Chile. 222 p.

Squeo F, G Arancio, J Gutiérrez (Eds.). 2001. Libro Rojo de la Flora Nativa y de los Sitios Prioritarios para su Conservación: Región de Coquimbo. La Serena, Chile. Ediciones Universidad de La Serena. 372 p.

Uribe M, R Durán, G Bravo, F Mora, P Cartes, C Delaveau. 2011. Propagación vegetativa de Berberidopsis corallina Hook.f., una especie en peligro de extinción, endémica de Chile. Gayana Botánica 68(2): 135-140.

Wells J. 1985 Plant propagation practices. Chicago, USA. American Nurseryman Publishing. 367 p.

Witte J, M Hall. 2004. Maderas del Sur de Chile. Árboles, Aplicaciones y Procesos. Segunda edición. Santiago, Chile. Editorial Universitaria. 98 p. 
